\title{
Making the Case for Modalities: The Need for Critical Thinking in Practice
}

\author{
Phil Page, PhD, PT, ATC, CSCS, FACSM ${ }^{1}$ \\ ${ }^{1}$ Franciscan University \\ Keywords: physical therapy, modalities, rehabilitation \\ https://doi.org/10.26603/001c.28326
}

\section{International Journal of Sports Physical Therapy}

Vol. 16, Issue 5, 2021

Physical therapists are playing an important role in the opioid epidemic by providing non-pharmacological pain control. For all the bad coming from this epidemic, there are silver linings. Kasiz et al. ${ }^{1}$ reported that direct access to physical therapy (PT) reduced the odds for short-term and long-term use of opioids in low back pain patients. The American Physical Therapy Association (APTA) campaign "Choose PT" encourages consumers to utilize PT for pain relief. ${ }^{2}$ Respondents to a Gallup poll in 2017 believed that PT was the safest and most effective treatment for lower back and neck pain. ${ }^{3}$ Not only are patients and providers recognizing the benefit of non-pharmacological pain relief, but we are learning more about pain and pain management through neuroscience research (more on that later).

Physical therapy interventions can include therapeutic exercise, manual therapy, patient education, and modalities. Yes, I said “modalities.” There's no doubt that there has been a backlash against modalities in recent years as more physical therapists demonize their use, particularly through social media. It seems the word "modalities" has become a taboo word, so much so that they are now referred to as "biophysical agents" in the APTA Guide to Physical Therapist Practice. ${ }^{4}$ The Guide to Practice offers a number of beneficial effects and indications for modalities (Table 1), which is where the irony begins: how can modalities (aka, biophysical agents) be so "bad" when our own Guide to Practice states so many intended benefit?

\section{CONFLICTING MESSAGES}

In 1995, the APTA House of Delegates took a stand against the exclusive use of biophysical agents/modalities (originally HOD PO6-95-29-18; amended in 2018 to HOD PO6-18-17-27 $)^{5}$ in response to the potential for overuse of modalities in physical therapy practice. Passive modality treatment became synonymous with the term "physical therapy" in other healthcare professions. Furthermore, the APTA contributed to the "Choosing Wisely" campaign in $2015,{ }^{6}$ advocating, “Don't use (superficial or deep) heat to obtain clinically important long term outcomes in musculoskeletal conditions." However, the explanation provided after that statement said, "While there is some evidence of short-term pain relief for heat, the addition of heat should be supported by evidence and used to facilitate an active treatment program." For some reason, that last statement supporting the use of heat for short-term pain relief as an
Table 1. Intended use for biophysical agents from the APTA Guide to Physical Therapist Practice. ${ }^{4}$

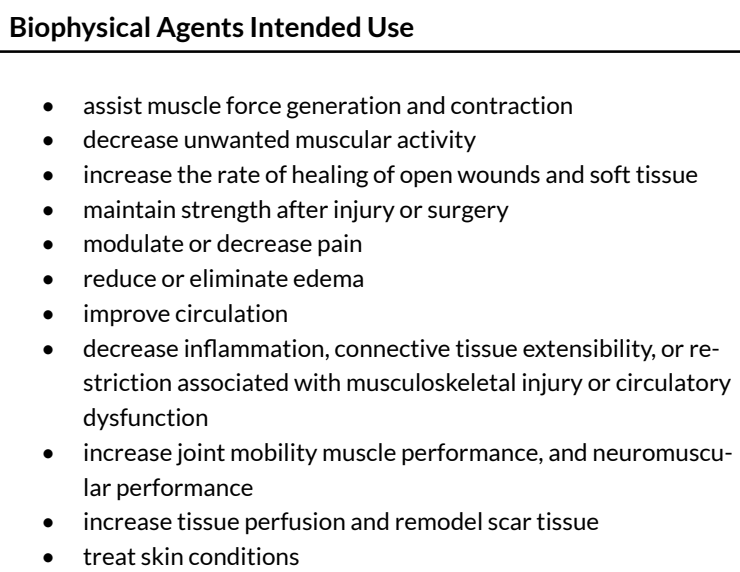

- assist muscle force generation and contraction

- decrease unwanted muscular activity

- increase the rate of healing of open wounds and soft tissue

- maintain strength after injury or surgery

- modulate or decrease pain

- reduce or eliminate edema

- improve circulation

- decrease inflammation, connective tissue extensibility, or restriction associated with musculoskeletal injury or circulatory dysfunction

- increase joint mobility muscle performance, and neuromuscular performance

- increase tissue perfusion and remodel scar tissue

- treat skin conditions

adjunctive in PT was lost by those decrying "modalities as malpractice.” Coupled with Medicare's withdrawal of reimbursement for superficial heat and cold around that time, the dominos had fallen against modalities in general.

Ironically, the APTA Guide to Physical Therapist Practice provides similar indications for manual therapy techniques as it does for biophysical agents (Table 2). ${ }^{4}$ In addition to pain relief, biophysical agents have more intended benefits than manual therapy such as facilitating muscle strength and tissue healing. Recognized as a "passive" treatment, manual therapy is almost always combined with another treatment such as therapeutic exercise or neuromuscular re-education and should be discontinued as the patient progresses in their rehabilitation. In this regard, manual therapy can be thought of as a short-term, passive adjunctive treatment to facilitate exercise...just as modalities should be considered as adjunctive in musculoskeletal rehabilitation. However, the use of manual therapy is not accompanied by the statement used with biophysical agents in the Guide: "The use of biophysical agents in the absence of other interventions should not be considered to be physical therapy unless there is documentation that justifies the necessity of their exclusive use."

The author of a recent article in the New York Times ${ }^{7}$ characterized modalities such as laser, ultrasound, and electrical stimulation as "voodoo treatments," suggesting 
Table 2. Comparison of the intended use of biophysical agents and manual therapy techniques, highlighting similar indications in bold font (From the APTA Guide to Physical Therapist Practice. ${ }^{4}$ )

Manual Therapy Technique Intended Use

- improve tissue extensibility

- increase range of motion

- induce relaxation

- mobilize or manipulate soft tissue and joints

- modulate pain

- reduce soft tissue swelling, inflammation, or restriction

Table 3. Grades of evidence used in Clinical Practice Guidelines from the Academy of Orthopedic Physical Therapy. 8

\begin{tabular}{|l|l|}
\hline $\begin{array}{l}\text { Grade of } \\
\text { Recommendation }\end{array}$ & Strength of Evidence \\
\hline A Strong evidence & $\begin{array}{l}\text { A preponderance of level I and or level II studies support the recommendation. This must include at least } \\
1 \text { level } 1 \text { study }\end{array}$ \\
\hline B Moderate evidence & $\begin{array}{l}\text { A single high-quality randomized controlled trial or a preponderance of level II studies support the } \\
\text { recommendation }\end{array}$ \\
\hline C Weak evidence & $\begin{array}{l}\text { A single level II study or a preponderance of level III and IV studies, including statements of consensus by } \\
\text { content experts, support the recommendation }\end{array}$ \\
\hline $\begin{array}{l}\text { D Conflicting } \\
\text { evidence }\end{array}$ & $\begin{array}{l}\text { Higher-quality studies conducted on this topic disagree with respect to their conclusions. The } \\
\text { recommendation is based on these conflicting studies }\end{array}$ \\
\hline $\begin{array}{l}\text { E Theoretical/ } \\
\text { foundational } \\
\text { evidence }\end{array}$ & $\begin{array}{l}\text { A preponderance of evidence from animal or cadaver studies, from conceptual models/ principles, or } \\
\text { from basic science/bench research support this conclusion }\end{array}$ \\
\hline $\begin{array}{l}\text { F Expert opinion } \\
\text { Best practice based on the clinical experience of the guidelines development team }\end{array}$ \\
\hline
\end{tabular}

that PT has a "lingering reputation for pseudoscience," further stating that "there is very little if any evidence that ultrasound does anything at all.” Once again, modalities played the "punching bag" for PT, further discrediting their use. The story's author suggests that physical therapists should use clinical practice guidelines (CPGs) to guide clinical decisions, noting favorable outcomes for exercise (Grade "A" evidence) in knee sprain patients, and less favorable (Grade "D") evidence for electrotherapy in treating plantar fasciitis patients. By cherry-picking these results, the New York Times author failed to note that electrotherapy has Grade A evidence in the Knee Sprain CPG. ${ }^{8}$ Of note, a Grade of $\mathrm{D}$ means there is conflicting evidence, not that the evidence is necessarily poor! (Table 3 ). Fueled by the call for "evidence-based practice," combined with bashing on social media, it seems modalities have been shoved to the other side of the pendulum with antiquated and shameful treatments.

\section{CLINICAL PRACTICE GUIDELINES SUPPORT USE AND NON-USE}

Clinical practice guidelines have been described as, “systematically developed statements to assist practitioner and patient decisions about appropriate health care for specific clinical circumstances." 9 While CPGs are highly regarded, they are still susceptible to bias and misinterpretation (as seen in the New York Times article). The authors of the CPG for patellofemoral pain (PFP) ${ }^{10}$ provided a "B" grade to support their recommendation against modality use, stating that "Clinicians should not use biophysical agents, including ultrasound, cryotherapy, phonophoresis, iontophoresis, electrical stimulation, and therapeutic laser, for the treatment of patients PFP." The recommendation was based on one 2011 systematic review of 12 low to moderate-quality studies using different modalities. ${ }^{11}$ However, in their explanation for the recommendations, the CPG authors stated, "There was no consistent evidence of any beneficial effect when a therapeutic modality was used alone.” Note that the CPG authors did not include the words "when used alone" from their overall recommendation against biophysical agents. A more recent systematic review agreed that no stand-alone intervention was effective in treating PFP patients ${ }^{12}$ however, there was some evidence that subgroups of PFP patients may benefit from different treatment modalities.

A review of 12 Academy of Orthopedic Physical Therapy (AOPT) $\mathrm{CPGs}^{13}$ revealed that biophysical agents are both recommended and not recommended for a variety of conditions at various grades of evidence (Table 4). It's clear that there are more evidence-supported uses for modalities in 
Table 4. Summary of grades of evidence with recommendations of modality use in orthopedic physical therapy CPGs. ${ }^{13}$

\begin{tabular}{|c|c|c|c|c|}
\hline \multirow{2}{*}{ Biophysical Agent } & \multicolumn{2}{|c|}{ YES: Recommended for use } & \multicolumn{2}{|c|}{ NO: Recommended against use } \\
\hline & Grade & Condition & Grade & Condition \\
\hline Biofeedback & B & Knee cartilage & B & Knee PFP \\
\hline \multirow{3}{*}{ Cryotherapy } & B & Knee ACL & \multirow{3}{*}{ B } & \multirow{3}{*}{ Knee PFP } \\
\hline & B & Knee TKA & & \\
\hline & C & Ankle sprains & & \\
\hline \multirow{3}{*}{ Diathermy } & $\mathrm{C}$ & Hand CTS & & \multirow{3}{*}{. } \\
\hline & C & Shoulder frozen & & \\
\hline & C & Ankle sprains & & \\
\hline \multirow{7}{*}{ Electrotherapy } & A & Knee ACL & \multirow{7}{*}{ B } & \multirow{7}{*}{ Knee PFP } \\
\hline & B & Knee cartilage & & \\
\hline & C & Hand CTS & & \\
\hline & C & Neck pain & & \\
\hline & $\mathrm{C}$ & Shoulder frozen & & \\
\hline & $\mathrm{D}$ & Ankle sprains & & \\
\hline & $\mathrm{D}$ & Foot plantar fasciitis & & \\
\hline Heat & C & Hand CTS & & \\
\hline \multirow{2}{*}{ lonotophoresis } & B & Ankle Achilles tendon & \multirow{2}{*}{ B } & \multirow{2}{*}{ Hand CTS } \\
\hline & $\mathrm{D}$ & Foot plantar fasciitis & & \\
\hline \multirow{3}{*}{ Laser } & C & Ankle sprains & B & Hand CTS \\
\hline & C & Foot plantar fasciitis & B & Knee PFP \\
\hline & D & Ankle Achilles tendon & & \\
\hline \multirow{2}{*}{ Phonophoresis } & C & Hand CTS & \multirow{2}{*}{ B } & \multirow{2}{*}{ Knee PFP } \\
\hline & C & Foot plantar fasciitis & & \\
\hline \multirow{5}{*}{ Ultrasound } & $\mathrm{B}$ & Нip OA & A & Ankle sprains \\
\hline & B & Knee PFP & C & Hand CTS* \\
\hline & $\mathrm{C}$ & Knee TKA & $\mathrm{C}$ & Foot plantar fasciitis \\
\hline & $\mathrm{C}$ & Shoulder frozen & & \\
\hline & D & Hand CTS* & & \\
\hline \multirow{2}{*}{ Mechanical Traction } & B & Neck pain & & \\
\hline & $\mathrm{D}$ & Back pain & & \\
\hline
\end{tabular}

See Table 3 for explanation of grades of evidence.

*The CTS CPG noted a grade of C against thermal ultrasound, but a grade of D in favor of non-thermal ultrasound

orthopedic CPGs than against; all CPGs recommended some type of modality. Only 4 (25\%) CPGs recommended against the use of modalities; most of the recommendations against the use of modalities in the one CPG for patellofemoral pain. ${ }^{10}$ Grade "A" or "B" recommendations for biophysical agents included biofeedback, cryotherapy, electrotherapy, iontophoresis, ultrasound, and mechanical traction. Obviously, there is evidence to support the use of certain modalities in certain patients; therefore, biophysical agents should be considered in specific patient populations.

A 2019 systematic review by Zadro and colleagues ${ }^{14}$ titled, "Do physical therapists follow evidence-based guidelines when managing musculoskeletal conditions?” aimed to compare evidence-based treatment recommendations to the percentage of physical therapy treatments reported in the literature. The authors reviewed 94 musculoskeletal evidence-based guidelines and systematic reviews for recommendations, comparing them to studies using utilization surveys of physical therapists and audits of musculoskeletal PT treatments. The authors reported that a median of $54 \%$ of physical therapists chose recommended treatments, while $43 \%$ choose treatments not recommended and $81 \%$ chose treatments without recommendations. In terms of biophysical agents, there appears to be a gap between musculoskeletal treatment recommendations and clinical practice. Only $28 \%(8 / 11)$ of recommendations were appropri- 
Table 5. Evidence-based recommendations for biophysical agents across different musculoskeletal conditions and the percentage of physical therapists reporting use. ${ }^{14}$ Over half of physical therapists reportedly do not follow recommendations for 8 out of 11 situations (72\%), noted by shading.

\begin{tabular}{|l|l|l|l|}
\hline \multirow{2}{*}{ Body Part } & Treatment & Recommended? & Percentage reporting use \\
\hline \multirow{4}{*}{ Low Back Pain } & Ultrasound/ electrotherapy & No & \multicolumn{2}{|l|}{} \\
\cline { 2 - 4 } & Heat & Yes & $39 \%$ \\
\hline \multirow{4}{*}{ Neck } & Heat/Cold & No & $53 \%$ \\
\cline { 2 - 4 } & Ultrasound/electrotherapy & No & $30 \%$ \\
\cline { 2 - 4 } & Laser & Yes & $6 \%$ \\
\hline \multirow{3}{*}{ Shoulder } & Electrotherapy & No & $90 \%$ \\
\cline { 2 - 4 } & Laser & Yes & $36 \%$ \\
\hline \multirow{2}{*}{ Knee } & Heat/Cold & Yes & $62 \%$ \\
\cline { 2 - 4 } & Ultrasound/electrotherapy & No & $43 \%$ \\
\hline \multirow{2}{*}{ Plantar Fasciitis } & Laser & Yes & $43 \%$ \\
\cline { 2 - 4 } & Ultrasound/electrotherapy & Yes & $43 \%$ \\
\hline
\end{tabular}

ately followed by at least $50 \%$ of physical therapists.

\section{A NEW LOOK AT THE EVIDENCE}

Modalities appear to be one of the most-researched interventions in physical therapy. A quick, informal search ${ }^{1}$ in PubMed in August 2021 resulted in 196,736 results for electrotherapy and 27,116 for ultrasound, as opposed to 158,786 results for exercise and 26,192 for manual therapy. While beyond the scope of this paper, there appears to be ample evidence to support the use of modalities for short-term pain relief and to promote a healing environment.

Several textbooks provide excellent summaries of the mechanisms behind modalities. ${ }^{15-17}$ Biophysical agents provide acute, short-term pain relief through direct and indirect mechanisms. Direct mechanisms include modulation of inflammatory mediators, slowed nerve conduction, pain signal blockage, and endogenous analgesia. In addition to pain relief, modalities offer direct therapeutic benefits through muscle stimulation and wound healing. Modalities are also thought to reduce pain indirectly through spasm reduction or through tissue healing, as evidenced by a supportive environment for wound healing increased blood flow. Unfortunately, much of the direct evidence on tissue healing with modalities is from experimental animal models; as, human subjects are not keen to volunteer for experimental crush injuries or ligament transections.

\section{OUTCOMES RESEARCH}

Some modality research outcomes focus on longer-term results; however, the natural process of healing of time may trump all factors. A common argument against modalities is the lack of impact on long-term outcomes; however, modalities are commonly recommended as an adjunct (during certain phases of rehabilitation) and offer short-term pain relief. Improvement in short-term goals has been associated with better outcomes in low back pain patients. ${ }^{18}$ Just as pain medications are recommended for short-term relief, modalities can provide acute, non-pharmaceutical pain relief. Continued use of multiple modalities beyond one to two weeks may not be necessary but might be considered in situations of sub-acute pain and exacerbations during a multi-modal rehabilitation program.

It is interesting to note that patient satisfaction is not always associated with better outcomes. ${ }^{19}$ A meta-analysis of patient satisfaction determinants ${ }^{20}$ reported that outpatient musculoskeletal patients seem equivocal to receiving passive treatments such as manual therapy or modalities, as compared to active exercise intervention, although some preferred exercise. The authors further noted that "an individualized approach to decision-making about treatment represented the best strategy to increase patient satisfaction." Providing modalities solely based on improving patient satisfaction may not warranted; rather, clinicians should include patients in the decision-making process when deciding if modalities are indicated to address specific

1 Note that these results only represent the number of studies published and do not represent quality or efficacy. PubMed search terms in August 2021 included, Search 1: “((physical therapy) OR (physiotherapy)) AND (electrotherapy) OR (electrical stimulation)”; Search 2: terms, "((physical therapy) OR (physiotherapy)) AND (ultrasound); Search 3: "((physical therapy) OR (physiotherapy)) AND (exercise) OR (therapeutic exercise)"; Search 4: "((physical therapy) OR (physiotherapy)) AND ((manual therapy) OR (manipulation) OR (manipulative therapy) OR (joint mobilization)) 
impairments and limitations.

Cost effectiveness research helps identify value of an intervention in healthcare systems by comparing outcomes and costs. A study from the United Kingdom found that modalities provided similar cost-benefit to manual therapy in a review of studies on patients with knee OA. ${ }^{21}$ In fact, they found TENS treatments to be the most cost-effective modality studied in knee OA patients. While some systematic reviews suggest electrotherapy modalities benefit some groups of patients, ${ }^{22,23}$ clinicians should consider the cost of these modalities in their clinical practice in respect to the types of patients they treat.

\section{NEUROSCIENCE RESEARCH}

Researchers and clinicians are helping us better-understand both mechanisms of pain and effective interventions. This evolving research expands on existing knowledge and offers new insights for potential non-pharmacological treatments. Emerging research from human and animal studies in pain science may provide information on other mechanisms and dose-responses for effective use of biophysical agents.

Relative to modalities, neuroscience research on pain control suggests other potential mechanisms for pain control. Thermal and electrical modalities are often thought to provide pain relief through the Gate Control Theory, where ascending pain signals in the peripheral nervous system are blocked at the spinal cord level. More recently, scientists have noted the influence of central nervous system descending signals in modulating pain. Researchers have shown painful stimuli can inhibit pain at a distant site through an endogenous mechanism in animal studies, known as "diffuse noxious inhibitory control" (DNIC) of pain. ${ }^{24}$ This is also a proposed pain relief mechanism of the "hurts so good" treatments such as roller massage and foam rolling. ${ }^{25}$

The DNIC mechanism is thought to play a role in chronic central sensitization of pain in patients with chronic pain such as knee osteoarthritis ${ }^{26}$ and fibromyalgia. ${ }^{27}$ Electroacupuncture has been studied in a mouse model, ${ }^{28}$ suggesting DNIC plays a role in knee osteoarthritis pain relief. In 2019, Peng et al. ${ }^{29}$ reported that acupuncture-like TENS influences pain through the descending pain inhibitory system from the prefrontal cortex in humans. Acupuncturelike TENS is considered a "noxious" stimulus rather than the more-comfortable conventional TENS and is often used to prevent nervous system adaptation particularly in chronic pain. The authors suggested that conventional TENS and acupuncture-like TENS mediate their analgesic effects through different mechanisms.

Biophysical agents are applied directly through the skin. Interestingly, the role of the skin in pain relief has little research. Fortunately, research in the integumentary and fascial systems and their role in pain modulation is slowly growing. Specialized receptors in the skin, transient receptor proteins (TRP) have been known to play a role in our sensation of temperature and may be activated by various substances in nature. For example, the TRP receptor for menthol (TRP-M8) is sensitive to both cold and menthol. More recently, researchers have discovered a potential role for TRP channels in pain modulation. ${ }^{30}$ The TRP-M8 re-

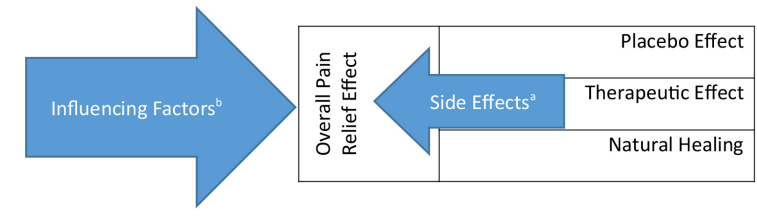

Figure 1. Internal contributions of natural healing, therapeutic effect, and placebo effect on overall pain relief. aSide effects of therapeutic agents should be considered as well. b ${ }^{b}$ Several external factors can influence the overall effect of a treatment such as adherence and compliance, contextual factors, therapeutic alliance, healing phase, previous injury, tissue health/viability, comorbidities, and risk behaviors.

ceptor has been shown to provide analgesia in rats with chronic neuropathies via the glutamate system in the spinal cord. ${ }^{31,32}$ Furthermore, Andersen et al. ${ }^{33}$ investigated the role of TRP-M8 in humans, providing in-vivo support for the Gate Control theory in pain and inflammation.

\section{EMBRACING THE PLACEBO EFFECT}

Some may feel that modalities offer little more than placebo effect. However, as discussed previously, evidence supports some level of therapeutic effect for biophysical agents. The placebo effect is only part of the overall effectiveness of an intervention (Figure 1). Several factors of a treatment contribute to overall pain relief. Natural healing provides a physiological effect on pain, while treatment provides both a therapeutic and placebo effect of varying degrees. Two treatments may have similar efficacy, but one may provide more therapeutic and less placebo effects (or vice versa). Some biophysical agents such as electrical stimulation may facilitate the natural healing process as well. Side effects may also play a role in the decision to use a specific treatment. As we learn more through pain research, the placebo effect has gained a new appreciation as a non-pharmacological pain relief mechanism with no side effects.

Beyond the therapeutic, placebo, and natural healing effects, other factors may play an important role in the overall effectiveness of a treatment. Better adherence and compliance are generally thought to positive treatment outcomes. Contextual factors and social determinants of health (SDH) such as socioeconomic status and education level may also influence outcomes. ${ }^{34} \mathrm{~A}$ therapeutic alliance (therapist-patient relationship) may enhance outcomes, ${ }^{35}$ although a strong relationship has not been established. ${ }^{36}$ Other factors such as the healing phase, previous injury, and tissue health may influence outcomes in addition to the presence of co-morbidities such as diabetes or risky behaviors such as smoking.

\section{DOSE RESPONSE}

Clinical trials often proceed through 3 phases of research. 
"Pre-clinical” studies are mechanistic bench studies (usually in animals) to evaluate the mechanism of action of a medication or treatment. Phase 1 clinical trials are first small groups of study participants to participate in the treatment. Phase 2 studies look at the dose-response to determine the appropriate amount of medication for a desired response (such as pain relief in humans), and Phase 3 trials are outcome studies in larger groups of patients, often involving placebos. Phase 2 studies are often lacking prior to outcomes research in non-pharmacological studies. Instead, most studies on these interventions evaluate outcomes prior to establishing a dose-response, which leads us to question ineffective treatment outcomes: was the appropriate dose of the treatment given for the desired outcome?

Many physical therapy interventions are limited by lack of a specific dose-response. The relationship of therapeutic dose (time, intensity, duration) and the titrated result on the desired response (pain, movement, swelling) remain unknown for many interventions and patient populations. Even the most recommended interventions such as manual therapy, therapeutic exercise and balance training lack specific dose-response parameters across patient populations. While the traditional " 3 sets of 10 " of an exercise is rooted in a physiological dose response to strengthening in healthy subjects, this dosage has not been well-evaluated in patient populations.

If a particular dose of an intervention such as ultrasound is being studied for efficacy, other questions arise from the research methodology. Devices should be calibrated prior to a study to ensure the prescribed dose is actually being administered; damage to ultrasound heads can go undetected, thus resulting in a lower or inaccurate dose. Furthermore, the technique of application can also hinder the appropriate dose from being delivered. "Textbook" application of ultrasound should be slow $(4 \mathrm{~cm} /$ second) and within a small area (twice the size of the sound head) to effectively deliver the energy to the tissue; ${ }^{15-17,37}$ yet many have witnessed a clinical application of ultrasound rarely stays within these parameters, thus affecting the intended dosage.

Some researchers have evaluated the dose-response of modalities as an adjunctive treatment. The "Stretching Window" was described by the late Dr. David Draper as the period following modality application to prepare tissues for active treatments such as stretching or mobilization. ${ }^{37}$ (Figure 2). Draper suggested that a $5^{\circ} \mathrm{C}$ increase in tissue temperature created by ultrasound would be effective for an average of 3.3 minutes after treatment. Adjunctive modalities provide a specific timeframe to acutely reduce pain or prepare tissue for active treatment. The length of this window varies depending on the modality, tissue type and depth of tissue. 38

\section{CONCERNING TRENDS}

Modalities aren't the only intervention that has become de-

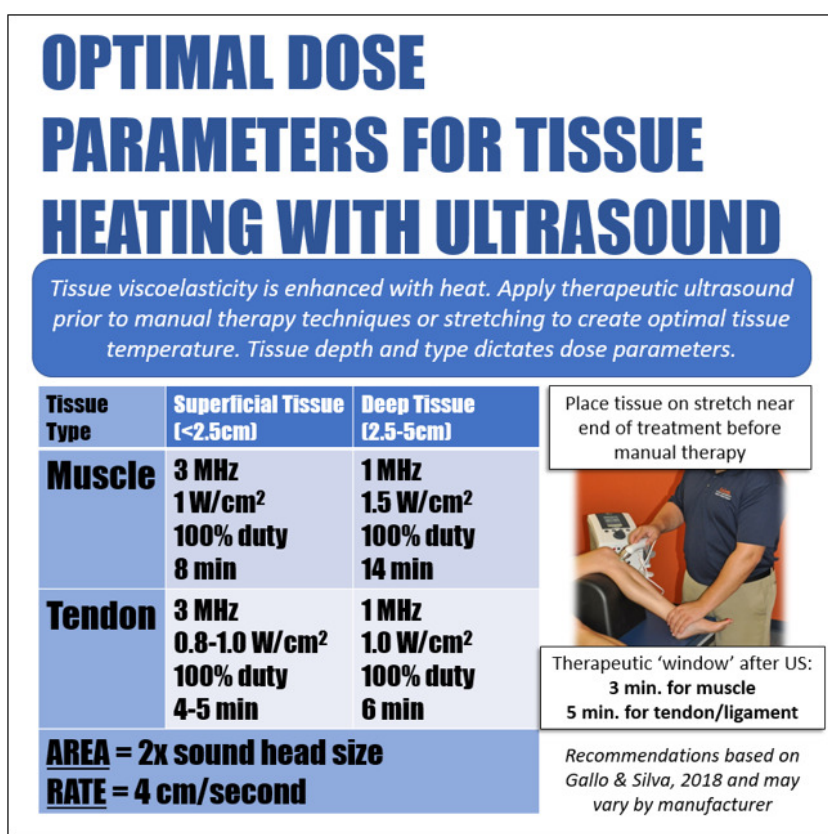

Figure 2. Recommendations for ultrasound dose parameters to heat tissues prior to stretching or mobilization as an adjunctive treatment, known as the "stretching window." 38

monized in PT; take isokinetics for example. In the 1970s and 80s, isokinetic testing and exercise were mainstays in outpatient orthopedic PT clinics. Despite evidence of its benefit, isokinetic devices became nearly extinct in the 1990s as reimbursement codes were removed and the cry for more "functional closed chain" exercises began (We'll save the discussion on today's epidemic of quadriceps weakness and ACL failure rates today for another time).

At the same time that isokinetics were waning, a new treatment was emerging: manual therapy. Manual therapy was heralded as a hands-on therapy applied to the patient with mechanisms best explained through physiological benefits to relieve pain and promote healing (sound familiar? See Table 2). Manual therapy quickly became the goldstandard in treating many orthopedic conditions, as is evidenced by their inclusion in nearly all AOPT CPGs with high grades of evidence. ${ }^{13}$

A concerning trend parallels the downfall of modalities and isokinetic exercise: "The Demonization of Manual Therapy," as recently published by Dr. Chad Cook. ${ }^{39}$ Some popular and effective interventions such as ultrasound become overutilized, while scientific evidence begins to cast doubt on the mechanism or efficacy of such treatments, which may lead to removal of reimbursement... oftentimes the deadly blow to an intervention. There's no doubt that interventions in physical therapy wax and wane in popularity as newer ${ }^{2}$ treatments emerge. The trend in the rise and

\footnotetext{
2 As an example, blood flow restriction therapy has been used since the 1960's in Japan, and even in the United States in the 1990's as an adjunct for exercise training particularly in the elderly. It wasn't until the late 2010's that blood flow restriction became a popular treatment in physical therapy, although it had been used for nearly 50 years prior.
} 
fall of popularity of interventions was originally described as "Scott's Parabola," 40 and was recently modified for physical therapy in an editorial in IJSPT. ${ }^{41}$ Clinicians sometimes abandon effective treatments for various reasons as new, less proven treatments become popular. Interestingly, this cycle seems to continue as a "roller coaster," where forgotten treatments regain their clinical popularity (sometimes slightly modified), such as rigid tape, electrical stimulation, or mechanical percussion.

The "Tomato Effect" has been used to explain this phenomenon as well. The Tomato Effect occurs "when an efficacious treatment for a certain disease is ignored or rejected because it does not 'make sense' in the light of accepted theories of disease mechanisms and drug actions." 42 Luckily, the Tomato Effect can be reversed as new evidence emerges that is consistent with accepted theories. Modalities are poised to see such a reversal as we have discussed previously with insights into clinical outcomes, pain neuroscience, dose-response, and the placebo effect.

\section{IT’S TIME FOR US TO ‘RE-THINK’ MODALITIES IN PHYSICAL THERAPY}

"Evidence-based" clinicians may re-evaluate their attitudes toward modalities, particularly since they are still included in the APTA Guide to Physical Therapist Practice, required in accredited DPT programs, and included as questions on the National PT Examination (NPTE). ${ }^{43}$ This requires critical appraisal skills to evaluate new research in context with existing evidence while considering limitations. While CPGs are excellent guides to assist evidence-based decision making, they are still limited to a small number of patient populations and should not be used to generalize decisions about other populations. Evidence-based practice does not rely on evidence alone; empirical experiences and patient values are integrated with the best evidence when determining which intervention is appropriate for each individual patient. Prescribing therapeutic modalities is a skilled intervention to correctly choose, dose, and apply as part of an individualized and integrated treatment approach.

The physiological benefits of modalities are well-established in bench studies and reported in several textbooks; ${ }^{15-17,37}$ however, these results don't always translate into human outcomes. There are clinical studies of varying quality reporting both for and against modality use in certain situations (as mentioned previously using electrotherapy as an example in CPGs). It's obvious that "one size does not fit all" when it comes to modalities; the recommendation for a specific modality remains specific to the population.

There is no argument that modalities have been overused and abused for financial gain by some, but the message should be loud and clear: Modalities are beneficial but should not be used as a stand-alone intervention. It's time to move on from stereotyping modalities as useless equipment and respect biophysical agents for all the benefits they provide, particularly as short-term non-pharmacological pain relief. It's time to stop bashing modality use on social media and recognize that there are legitimate applications of ultrasound, laser, and cryotherapy that are supported with high-level clinical practice guidelines. Are we using modalities as intended and indicated, or are we denying patients a beneficial modality? Don't throw the baby out with the bathwater. Re-think the evidence as new research evolves. Recognize legitimate use of modalities to reduce pain and support a healing environment when used as an adjunct to active treatment, particularly as an alternative to pharmacological management.

Submitted: August 27, 2021 CDT, Accepted: September 20, 2021 CDT 


\section{REFERENCES}

1. Kazis LE, Ameli O, Rothendler J, et al.

Observational retrospective study of the association of initial healthcare provider for new-onset low back pain with early and long-term opioid use. BMJ Open. 2019;9(9):e028633. doi:10.1136/bmjopen-2018-02863 $\underline{3}$

2. American Physical Therapy Association. ChoosePT. https://www.choosept.com/Default.aspx. Accessed September 3, 2021.

3. Gallup.com. Americans Prefer Drug-Free Pain Management Over Opioids. Gallup.com. https://new s.gallup.com/reports/218495/s.aspx.

4. American Physical Therapy Association. APTA Guide to Physical Therapist Practice Categories of Interventions. https://guide.apta.org/interventions/c ategories-interventions.

5. American Physical Therapy Association. Exclusive use or use of multiple biophysical agents HOD P06-18-17-27. https://www.apta.org/siteassets/pdfs/p olicies/exclusive-use-or-use-of-multiple-biophysicalagents.pdf.

6. ABIM Foundation. Choosing Wisely. American Physical Therapy Association.

7. Smith D. What to look for in a physical therapist. New York Times. July 6, 2021.

8. Logerstedt DS, Scalzitti D, Risberg MA, et al. Knee Stability and Movement Coordination Impairments: Knee Ligament Sprain Revision 2017. J Orthop Sports Phys Ther. 2017;47(11):A1-A47. doi:10.2519/jospt.201 $\underline{7.0303}$

9. Institute of Medicine. Clinical Practice Guidelines: Directions for a New Program. Washington DC: National Academy Press; 1990. doi:10.17226/1626

10. Willy RW, Hoglund LT, Barton CJ, et al. Patellofemoral Pain. J Orthop Sports Phys Ther. 2019;49(9):CPG1-CPG95. doi:10.2519/jospt.2019.030 $\underline{2}$

11. Lake DA, Wofford NH. Effect of therapeutic modalities on patients with patellofemoral pain syndrome: a systematic review. Sports Health. 2011;3(2):182-189. doi:10.1177/1941738111398583
12. Saltychev M, Dutton RA, Laimi K, Beaupre GS, Virolainen P, Fredericson M. Effectiveness of conservative treatment for patellofemoral pain syndrome: A systematic review and meta-analysis. $J$ Rehabil Med. 2018;50(5):393-401. doi:10.2340/165019 $\underline{77-2295}$

13. Page P, Mistretta C, Thompson J, Brittain K. Musculoskeletal clinical practice guidelines recommended therapeutic interventions. (Abstract). Poster Presentation presented at the: American Physical Therapy Association-Louisiana; August 27, 2021; Baton Rouge, LA.

14. Zadro J, O’Keeffe M, Maher C. Do physical therapists follow evidence-based guidelines when managing musculoskeletal conditions? Systematic review. BMJ Open. 2019;9(10):e032329. doi:10.1136/b miopen-2019-032329

15. Cameron MH. Physical Agents in Rehabilitation. 5th ed. Saunders; 2018.

16. Bellew JWM SL, Nolan TP. Michlovitz's Modalities for Therapeutic Intervention. 6th ed. F.A. Davis; 2016.

17. Draper DOJ LS, Knight KL. Therapeutic Modalities. The Art and Science. 3rd ed. Philadelphia, PA: Wolters Kluwer; 2021.

18. Walston Z, McLester C. Importance of early improvement in the treatment of low back pain with physical therapy. Spine (Phila Pa 1976). 2020;45(8):534-540. doi:10.1097/BRS.0000000000003 $\underline{318}$

19. Hush JM, Cameron K, Mackey M. Patient satisfaction with musculoskeletal physical therapy care: a systematic review. Phys Ther. 2011;91(1):25-36. doi:10.2522/ptj.20100061

20. Rossettini G, Latini TM, Palese A, et al. Determinants of patient satisfaction in outpatient musculoskeletal physiotherapy: a systematic, qualitative meta-summary, and meta-synthesis. Disabil Rehabil. 2020;42(4):460-472. doi:10.1080/0963 $\underline{8288.2018 .1501102}$

21. Woods B, Manca A, Weatherly H, et al. Costeffectiveness of adjunct non-pharmacological interventions for osteoarthritis of the knee. PLoS One. 2017;12(3):e0172749. doi:10.1371/journal.pone.0172 $\underline{749}$ 
22. Page MJ, Green S, Mrocki MA, et al.

Electrotherapy modalities for rotator cuff disease. Cochrane Database Syst Rev. 2016;(6):CD012225. doi:1 0.1002/14651858.CD012225

23. Page MJ, Green S, Kramer S, Johnston RV, McBain B, Buchbinder R. Electrotherapy modalities for adhesive capsulitis (frozen shoulder). Cochrane Database Syst Rev. 2014;(10):CD011324. doi:10.1002/ 14651858.CD011324

24. Le Bars D, Villanueva L, Bouhassira D, Willer JC. Diffuse noxious inhibitory controls (DNIC) in animals and in man. Patol Fiziol Eksp Ter. 1992;(4):55-65.

25. Aboodarda SI, Spence AJ, Button DC. Pain pressure threshold of a muscle tender spot increases following local and non-local rolling massage. BMC Musculoskelet Disord. 2015;16:265. doi:10.1186/s1289 1-015-0729-5

26. Arendt-Nielsen L, Nie H, Laursen MB, et al. Sensitization in patients with painful knee osteoarthritis. Pain. 2010;149(3):573-581. doi:10.101 6/i.pain.2010.04.003

27. Sumpton JE, Moulin DE. Fibromyalgia. Handb Clin Neurol. 2014;119:513-527. doi:10.1016/B978-0-702 0-4086-3.00033-3

28. Yuan XC, Zhu B, Jing XH, et al. Electroacupuncture Potentiates Cannabinoid Receptor-Mediated Descending Inhibitory Control in a Mouse Model of Knee Osteoarthritis. Front Mol Neurosci. 2018;11:112. doi:10.3389/fnmol.2018.00112

29. Peng WW, Tang ZY, Zhang FR, et al. Neurobiological mechanisms of TENS-induced analgesia. Neuroimage. 2019;195:396-408. doi:10.101 6/j.neuroimage.2019.03.077

30. Gonzalez-Ramirez R, Chen Y, Liedtke WB, Morales-Lazaro SL. TRP Channels and Pain. In: Emir TLR, ed. Neurobiology of TRP Channels. Frontiers in Neuroscience; 2017:125-147.

31. Proudfoot CJ, Garry EM, Cottrell DF, et al. Analgesia mediated by the TRPM8 cold receptor in chronic neuropathic pain. Curr Biol. 2006;16(16):1591-1605. doi:10.1016/j.cub.2006.07.06 1

32. Kumamoto E, Fujita T, Jiang CY. TRP Channels Involved in Spontaneous L-Glutamate Release Enhancement in the Adult Rat Spinal Substantia Gelatinosa. Cells. 2014;3(2):331-362. doi:10.3390/cell $\underline{\text { s3020331 }}$
33. Andersen HH, Gazerani P, Arendt-Nielsen L. HighConcentration L-Menthol Exhibits Counter-Irritancy to Neurogenic Inflammation, Thermal and Mechanical Hyperalgesia Caused by Transcinnamaldehyde. J Pain. 2016;17(8):919-929. doi:10.1 016/j.jpain.2016.05.004

34. Rethorn ZD, Cook C, Reneker JC. Social determinants of health: if you aren't measuring them, you aren't seeing the big picture. J Orthop Sports Phys Ther. 2019;49(12):872-874. doi:10.2519/jospt.2019.06 $\underline{13}$

35. Kinney M, Seider J, Beaty AF, Coughlin K, Dyal M, Clewley D. The impact of therapeutic alliance in physical therapy for chronic musculoskeletal pain: A systematic review of the literature. Physiother Theory Pract. 2020;36(8):886-898. doi:10.1080/09593985.201 8.1516015

36. Taccolini Manzoni AC, Bastos de Oliveira NT, Nunes Cabral CM, Aquaroni Ricci N. The role of the therapeutic alliance on pain relief in musculoskeletal rehabilitation: A systematic review. Physiother Theory Pract. 2018;34(12):901-915. doi:10.1080/09593985.20 18.1431343

37. Draper DO, Ricard MD. Rate of temperature decay in human muscle following $3 \mathrm{mhz}$ ultrasound: the stretching window revealed. J Athl Train. 1995;30(4):304-307.

38. Gallo JA, Silva KJ. Dosing Therapeutic Ultrasound to Induce Vigorous Heating Prior to Stretching and Manual Therapy The First Aider. Gardner, Kansas: Cramer Sports Medicine; 2018.

39. Cook CE. The demonization of manual therapy. Muskuloskelettale Physiotherapie. 2021;25:125-132.

40. Scott JW. Scott's parabola. BMJ. 2001;323(7327):1477. doi:10.1136/bmj.323.7327.1477

41. Page P. The Need for Critical Thinking in Rehabilitation Research. Int J Sports Phys Ther. 2021;16(4):27146. doi:10.26603/001c.27146

42. Goodwin JS, Goodwin JM. The tomato effect. Rejection of highly efficacious therapies. JAMA. 1984;251(18):2387-2390. doi:10.1001/jama.251.18.23 $\underline{87}$

43. Therapy FoSBoP. NPTE-PT Test Content Outline. https://www.fsbpt.org/Portals/0/documents/free-reso urces/ContentOutline 2018PTT 20170126.pdf?ver=1N O3y_U8T78U5uSen4H7vg\%3d\%3d. Accessed September 12, 2021. 